\title{
Interaction-enhanced flow of a polariton superfluid current in a ring
}

\author{
A. Gallemí,,${ }^{1,2,3}$ M. Guilleumas, ${ }^{1,4}$ M. Richard, ${ }^{5}$ and A. Minguzzi ${ }^{6}$ \\ ${ }^{1}$ Departament de Física Quàntica i Astrofísica, Universitat de Barcelona, E-08028 Barcelona, Spain \\ ${ }^{2}$ Institut de Nanociència i Nanotecnologia de la Universitat de Barcelona, IN ${ }^{2}$ UB, E-08028 Barcelona, Spain \\ ${ }^{3}$ INO-CNR BEC Center and Dipartimento di Fisica, Università degli Studi di Trento, 38123 Povo, Italy \\ ${ }^{4}$ Institut de Ciències del Cosmos, Universitat de Barcelona, ICCUB, 08028-Barcelona, Spain \\ ${ }^{5}$ Univ. Grenoble Alpes, and CNRS, Institut Néel, 38000 Grenoble, France \\ ${ }^{6}$ Univ. Grenoble Alpes, and CNRS, LPMMC, 38000 Grenoble, France
}

(Received 24 July 2017; revised manuscript received 22 June 2018; published 5 September 2018)

\begin{abstract}
We study the quantum hydrodynamical features of exciton polaritons flowing circularly in a ring-shaped geometry. We consider a resonant-excitation scheme in which the spinor polariton fluid is set into motion in both components by spin-to-orbital angular momentum conversion. We show that this scheme allows us to control the winding number of the fluid and to create two circulating states differing by two units of the angular momentum. We then consider the effect of a disorder potential, which is always present in realistic nanostructures. We discuss how a smooth disorder can be efficiently screened by the polariton-polariton interactions, yielding a signature of polariton superfluidity. This effect is reminiscent of supercurrent in a superconducting loop.
\end{abstract}

DOI: 10.1103/PhysRevB.98.104502

\section{INTRODUCTION}

Superfluidity is a striking feature of quantum fluids. It is characterized by an irrotational particle flow, which is frictionless below a certain critical velocity. Superflow is a typical manifestation of a superfluid: When the latter is trapped in a ring and set into circular motion, it will exhibit (i) an integer angular momentum in units of $\hbar$ and (ii) a vanishing decay of the current. This phenomenon has been observed a long time ago in a superconducting loop below the critical current [1], with superfluid helium [2] and more recently in ultracold atom condensates [3].

Exciton polaritons, in spite of their nonequilibrium character, have also been found to display many features of superfluidity, like frictionless flow [4], quantized vortices [5-7], and Bogoliubov-like dispersion [8]. A specific feature of polaritons is the fact that the superfluid can be excited resonantly both in terms of phase and amplitude. As a result, nontrivial flow patterns with finite angular momentum have been imprinted and studied [9]. Moreover, polaritons benefit from a spin-orbit coupling allowing for spin-to-orbital angular momentum conversion [10].

Ring-shaped confining geometries are very favorable for studying superfluidity: The periodic boundary conditions imposed by the ring allow for the study of supercurrent flows and verify quantized circulation, characterizing the irrotational velocity field of the superfluid. In this work, we examine theoretically the polaritonic superfluid current in a ring trap. Polaritonic microcavities etched into complicated shapes, such as rings, can be experimentally realized nowadays with a high degree of accuracy using state-of-the-art semiconductor nanotechnology [11-13]. In order to excite the circular motion of the polariton fluid, we rely on the specific spin-to-orbital angular momentum conversion mechanism. As a result, the angular momentum achieved by the polariton fluid is not directly imprinted by the excitation laser phase pattern.

Semiconductor nanostructures are often characterized by some degree of unavoidable spatial disorder. These features have to be carefully taken into account in the study of polariton flows: In the presence of disorder, Bose fluids are subject to localization, i.e., Anderson localization for vanishing interaction [14] or many-body localization in the strongly interacting case $[15,16]$. These localization mechanisms hinder the quantum fluid flow. However, repulsive interactions also screen the disorder experienced by the fluid, which on the contrary, helps restoring the flow, such that the net effect of interactions in the presence of disorder is in general not easy to determine. Note that such a screening effect has been reported already in a disordered polariton condensate [17] and in ultracold atoms in harmonic traps [18] (see, e.g., Ref. [19] for a comprehensive review). The effect of disorder on persistent currents has been the object of intense studies for fermionic systems, for negligible interactions (see, e.g., Ref. [20], and references therein), as well as including interaction effects [21].

In this work, we examine the case of a bosonic quantum fluid in driven-dissipative conditions confined within a ringshaped trap of finite thickness, and we investigate the competition between this angular momentum generation mechanism, and the backscattering due to disorder within the ring. We show that while the buildup of a net polariton flow (i.e., angular momentum) by spin-to-orbital angular momentum conversion is prevented at large disorder amplitude and weak polariton-polariton interaction, it is restored by increasing the interactions.

The paper is organized as follows. In Sec. II we introduce the model that describes the polarization-dependent polariton field, including the transversal electric-transversal magnetic (TE-TM) splitting which is present in realistic polaritonic 
microstructures. Section III describes the mechanism of spinto-orbital angular momentum conversion using numerical simulations of the coupled driven-dissipative Gross-Pitaevskii equations for the ring-trapped condensate. In Sec. IV, the interplay between interactions and disorder is analyzed, and the suppression of supercurrents is estimated. Finally, in Sec. V we summarize our results and discuss perspectives.

\section{THE MODEL}

Polaritons are bosonic quasiparticles of mixed excitonphoton nature that exist in semiconductor microcavities in the strong coupling regime [22]. In this work, we consider the lower polariton state, the dispersion of which is well described by a two-coupled harmonic oscillator model as $E_{\mathrm{pol}}=\frac{1}{2}\left(\hbar \omega_{c}(k)+E_{\mathrm{x}}\right)-\frac{1}{2} \sqrt{\left(\hbar \omega_{c}(k)-E_{\mathrm{x}}\right)^{2}+4 \Omega^{2}}$, where $\Omega$ is the exciton-photon Rabi splitting, $E_{\mathrm{x}}$ is the exciton energy, and $\hbar \omega_{c}(k) \simeq E_{\mathrm{c}}+\hbar^{2} k^{2} / 2 m_{\text {eff }}$ is the bare cavity photon dispersion characterized by an effective mass $m_{\text {eff }}$ that typically amounts to $10^{-5}$ in free electron mass units, and $E_{\mathrm{c}}$, which is the photonic zero-point kinetic energy.

In the following, we include the cavity photon polarization degree of freedom in our description in terms of a pseudospin by means of the components of the Stokes vector. Our discussion will involve two polarization basis: the circular polarization basis $| \pm\rangle$ relevant to polariton-polariton interactions and the horizontal-vertical linear polarization basis $|h\rangle,|v\rangle$ which is important in order to account for the TE-TM splitting of the cavity mode. The two basis are related by a rotation according to the usual transformation $| \pm\rangle=(|h\rangle \pm i|v\rangle) / \sqrt{2}$.

Indeed, owing to the Fresnel relation, TE and TM polarized light experience a slightly different optical path within the cavity, which gives rise to a slightly different effective mass $m_{\mathrm{TE}}, m_{\mathrm{TM}}$ for both polarization states. Figure 1(a) shows the TE-TM splitting [23] versus wave vector $k$, with the following parameters: $m_{\mathrm{TE}}=1.94 \times 10^{-5} m_{\mathrm{e}}, m_{\mathrm{TM}}=2.06 \times 10^{-5} m_{\mathrm{e}}$, $E_{\mathrm{c}}=2750 \mathrm{meV}, E_{\mathrm{x}}=2820 \mathrm{meV}$, and $\Omega=30 \mathrm{meV}$. These parameters have been chosen as to match ZnSe-based microcavities with which we plan to implement an experimental realization of this proposal.

In the simulations that we will present in the following sections, the radial momenta are discretized due to the confinement within the ring. The TE and TM modes, having a different effective mass, are thus split. We maximize the effect of the TE-TM splitting by applying a radial momentum $k_{p}=5.2 \mu \mathrm{m}^{-1}$ to the pump beam.

We now define $\psi_{\alpha}(\vec{r}, t)$ as the polariton field with a polarization state $\alpha$. The corresponding dynamics is determined by two coupled driven-dissipative Gross-Pitaevskii equations [24]. In the circular polarization basis it reads:

$$
\begin{aligned}
i \hbar \frac{\partial}{\partial t} \psi_{\alpha}(\vec{r}, t)= & {\left[T_{\alpha \alpha}^{\operatorname{circ}}(\vec{k})+V(\vec{r})-i \hbar \gamma_{\alpha}\right] \psi_{\alpha}(\vec{r}, t) } \\
& +T_{\alpha \beta}^{\mathrm{circ}}(\vec{k}) \psi_{\beta}(\vec{r}, t)+\left(g_{\alpha \alpha}\left|\psi_{\alpha}(\vec{r}, t)\right|^{2}\right. \\
& \left.+g_{\alpha \beta}\left|\psi_{\beta}(\vec{r}, t)\right|^{2}\right) \psi_{\alpha}(\vec{r}, t)+i E_{\alpha}^{\text {field }}(\vec{r}, t),
\end{aligned}
$$

where $T_{\alpha \beta}^{\mathrm{circ}}(\vec{k})$ is the kinetic tensor in the circular polarization basis, $V(\vec{r})$ is an external potential, which includes the ring
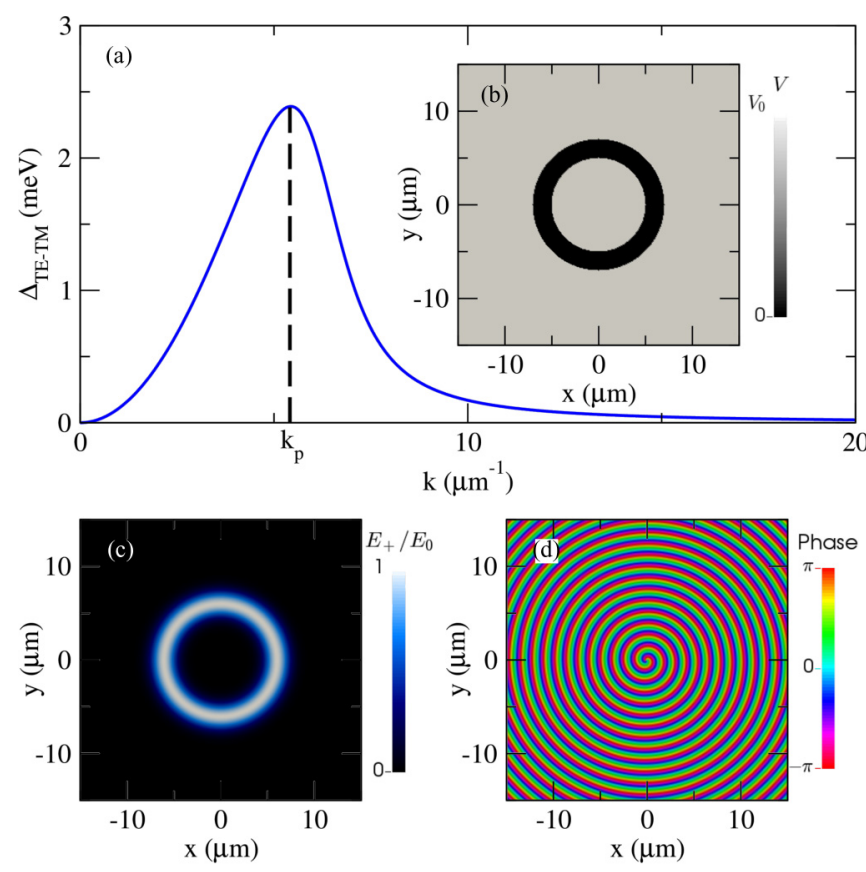

FIG. 1. Panel (a): TE-TM splitting as a function of the wave vector. The dashed line points the value of the wave vector of the pump chosen in the simulations, i.e., $k_{p}=5.2 \mu \mathrm{m}^{-1}$. Panel (b): ring-trap potential used in Sec. III. Panels (c) and (d): intensity and phase of the pump, respectively, for a winding number of the pump equal to 1 .

confinement and an optional disorder potential, and $g_{\alpha \alpha}\left(g_{\alpha \beta}\right)$ is the intercomponent (intracomponent) interaction strength. The subindices $\alpha, \beta=+,-; \alpha \neq \beta$ describe the different polarization components of the polariton field. The drivendissipative features are explicitly included by means of the loss rate $\gamma_{\alpha}=1 / \tau_{\alpha}$ describing polaritons leaking throughout the microcavity mirrors, where $\tau_{\alpha}=2 \mathrm{ps}$ is the polariton lifetime and a coherent pump term $E_{\alpha}^{\text {field }}(\vec{r}, t)$ that injects polaritons resonantly.

For the kinetic tensor, we use [24]

$$
T^{\mathrm{circ}}(\vec{k})=\left(\begin{array}{cc}
\hbar \omega & \Delta \frac{\left(-k_{x}+i k_{y}\right)^{2}}{k^{2}} \\
\Delta \frac{\left(-k_{x}-i k_{y}\right)^{2}}{k^{2}} & \hbar \omega
\end{array}\right),
$$

where $\hbar \omega=\left(\hbar \omega_{\mathrm{TM}}+\hbar \omega_{\mathrm{TE}}\right) / 2$ is the average energy between the TE and the TM cavity modes, and $\Delta=\hbar\left(\omega_{\mathrm{TM}}-\omega_{\mathrm{TE}}\right) / 2$ is the TE-TM splitting. Notice that the off-diagonal terms effectively play the role of a spin-orbit coupling.

\section{SPIN-TO-ORBITAL ANGULAR MOMENTUM CONVERSION}

One of the most striking effects that arise from the TE-TM splitting is the possibility to generate vortices by effective spin-orbit coupling, which leads to a spin-to-orbital angular momentum (SOAM) conversion. It means that we can excite a polaritonic field of a given polarization components with zero-angular momentum and obtain a vortex with winding number two in the cross-polarized component. This effect was theoretically predicted in Ref. [25] and experimentally 
confirmed in Ref. [10] in a homogeneous two-dimensional semiconductor. We present an alternative derivation of this effect in Appendix. In this section, we analyze this effect in a ring-shaped trap for polaritons. In this case, the vortex appears as a quantized azimuthal current flow along the ring, which is reminiscent of a persistent current of a superfluid within a loop.

\section{Spin-to-orbital angular momentum conversion in ring-shaped traps}

We use the following potential to describe the ring-shaped trap in the driven-dissipative Gross-Pitaevskii equation (1):

$$
V(r)=V_{0}\left(1-\frac{\sinh (w / \xi)}{\cosh (w / \xi)+\cosh \left(\left(r-R_{0}\right) / \xi\right)}\right),
$$

where $r$ is the two-dimensional radial coordinate. This potential corresponds to a ring with mean radius $R_{0}=6 \mu \mathrm{m}$, width $w=1 \mu \mathrm{m}$, and depth $V_{0}=1 \mathrm{eV}$. The profile of the edges of the trap is described by the parameter $\xi$, which we fix to be $w / 10$. The potential is represented in Fig. 1(b).

The pump geometry is illustrated in panels (c) and (d) of Fig. 1 for the intensity and the phase, respectively: It consists of a ring-shaped amplitude with a radial phase dependence $k_{p} r$, plus an azimuthal phase dependence $q \theta$ that imprints a certain angular momentum with winding number $q$. The expression of the pump then reads:

$$
E_{+}^{\text {field }}(r, \theta)=E_{0} e^{-\frac{\left(r-R_{0}\right)^{2}}{2 w^{2}}} e^{i k_{p} r} e^{i q \theta},
$$

where $E_{0}=10 \mathrm{meV} / \mu \mathrm{m}$ is the amplitude of the pump. We choose a $\sigma^{+}$polarized pump, whose intensity distribution matches the shape of the ring. In addition, it can imprint orbital angular momentum to polaritons, in the same way as in Ref. [7], where the authors used this property to generate a vortex. This type of pump profile could be experimentally realized, e.g., by using a spatial light modulator, or by phase plates, used to convert a standard laser mode into a vortex mode.

We have numerically solved the two-dimensional drivendissipative Gross-Pitaevskii equation (1) to obtain the steady state of the system. In Fig. 2 we plot the phase profile of the polariton field of the $\sigma^{+}$(top row) and the $\sigma^{-}$(bottom row) component inside the ring (i.e., for $\left|r-R_{0}\right|<w$, where the density does not vanish). The figure shows clearly quantization of circulation, i.e., one of the main features of superfluid flow, as can be readily obtained by the estimation of the winding number $q$ from the relation

$$
\oint \vec{v}_{s} \cdot d \vec{\ell}=2 \pi q \hbar / m
$$

where the superfluid velocity is related to the condensate phase $\phi(\vec{r})$ by $\vec{v}_{s}=\hbar \vec{\nabla} \phi(\vec{r}) / m$. Figure 2 shows in particular the case where the winding number of the angular momentum carried by the pump is $q=-1$ in the left column, $q=0$ in the middle column, and $q=1$ in the right column. As expected, in the stationary state, the component copolarized with the pump exhibits a superfluid current with a phase winding
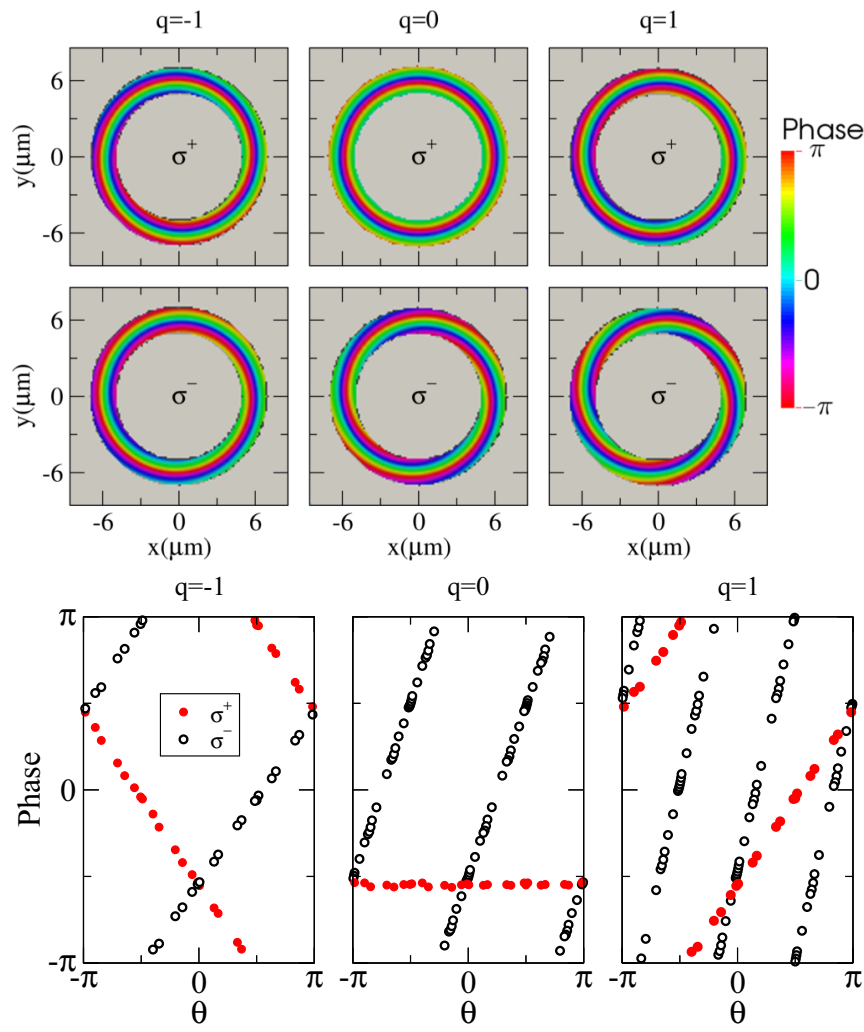

FIG. 2. Top view of the phase profile of the $\sigma^{+}$(top row) and the $\sigma^{-}$(middle row) components, in the steady state for different values of the winding number imprinted by the pump. In the left panels, $q=-1$, in the middle panels, $q=0$, and in the right panels, $q=1$. The winding number of the supercurrent nucleated in the $\sigma^{-}$component is two units larger than that of the pump. Bottom row: sections of the phase profile of the $\sigma^{+}$(red filled circles) and $\sigma^{-}$(black open circles) components as a function of the angle $\theta$ along the ring, at $r=R_{0}$, for the same choice of pump winding number.

number matching the pump. Interestingly, we find that the cross-polarized component exhibits a superfluid current with winding number $q+2$, i.e., a two-units increase with respect to the winding number of the pump. This is consistent with what is expected for a homogeneous system (see Appendix). It is also interesting to notice a large radial component of the phase gradient in each ring, resulting from the spin-orbit coupling between the two spin components. Correspondingly, we find a modulation in the radial density profile of each component, which is due to the coupling between the transverse modes.

The spin-to-orbital angular momentum conversion can be also seen in the bottom row of Fig. 2, where we show the phase $\phi\left(r=R_{0}, \theta\right)$ of the $\sigma^{+}$polarized field (red filled circles) and of the $\sigma^{-}$(black open circles) one in the steady state. The winding number of the pump is $q=-1$ (left panel), $q=0$ (middle panel), and $q=1$ (right panel). We see that the phase of the component winds by $2 \pi, 4 \pi$, and $6 \pi$, respectively, i.e., in the $\sigma^{-}$component, the winding number is $q=1,2$, and 3 as expected from the middle row panels in Fig. 2. 


\section{POLARITON CURRENT: COMPETITION BETWEEN DISORDER AND INTERACTIONS}

In the previous section, we have shown that when we excite the $\sigma^{+}$polariton component into a mode carrying no angular momentum in a smooth ring-shaped trap, the $\sigma^{-}$component acquires a superfluid current with winding number 2 . In this section we account for the fact that in realistic experiments, a (gaussian-distributed) disorder potential experienced by polaritons is present within the ring,

$$
V_{\text {dis }}(\vec{r})=\operatorname{Re}\left[\mathcal{F}^{-1}\left[2 \pi^{2} l_{c} U_{0} \exp \left(i \varphi_{\vec{k}}\right) \exp \left(-k^{2} l_{c}^{2} / 4\right)\right](\vec{r})\right],
$$

where $U_{0}$ is the strength of the disorder, $l_{c}$ is the correlation length, which gives the order of magnitude of the distance between maxima and minima of the disorder potential, and $\varphi_{\vec{k}}$ is a random matrix with phases uniformly distributed between 0 and $2 \pi$. We analyze the interplay of the disorder strength and interactions on the polariton current along the ring. Changes in the correlation length, which we fix to be $l_{c}=1.5 \mu \mathrm{m}$ in this work, may lead to a localization-delocalization transition, as recently shown in 2D [26] and 3D [27]. However, we are interested in the study of the dependence of the mobility edge as a function of interactions, and the study in terms of correlation length is beyond the scope of the paper.

In the simulations, we have chosen the typical situation in which the polariton-polariton interaction is ten times larger in the copolarized case than in the cross-polarized one, and of opposite sign, i.e., we take $g=g_{++}=g_{--}=-10 g_{+-}$, in agreement with theoretical and experimental studies [28-31]. We use the pump to excite the $\sigma^{+}$component with a pump beam with $q=0$ orbital angular momentum. To monitor the superfluid current induced in the $\sigma^{-}$component, we compute the expectation value of the angular momentum operator around the $z$ axis $\mathcal{L}_{z}=\left\langle\hat{L}_{z}\right\rangle$, normalized to its maximum value.

In the top panel of Fig. 3, we show the angular momentum computed from the average of ten simulations performed with different realizations of the disorder potential, as a function of the interaction strength $g$ and disorder strength $U_{0}$. We can see from the figure that there is a (red) region in which the disorder can be simply neglected, since it does not affect the polariton field, the current is preserved and the system remains superfluid. However, as the disorder strength increases above a given critical value (yellow region), the polariton supercurrent diminishes. Moreover, larger interactions require larger values of the disorder strength in order to observe the decrease of such a current. The reason of this effect is the fact that the disorder is efficiently screened by the interactions. On the contrary, the polariton current is suppressed as disorder overcomes interaction. We have also checked that, for our choice of copolarized interaction parameters, the magnitude of angular momentum is only weakly affected by the crosspolarized interaction strength, and similar results to those shown in Fig. 3 are found for the choice $g=g_{++}=g_{--}=$ $10 g_{+-}$.

For each point of the top panel, we have computed the standard deviation $\sigma_{\mathcal{L}}$ of the different values of $\mathcal{L}_{z}$ obtained for each realization of the disorder. The result is represented in the middle panel of Fig. 3. We see that when a current
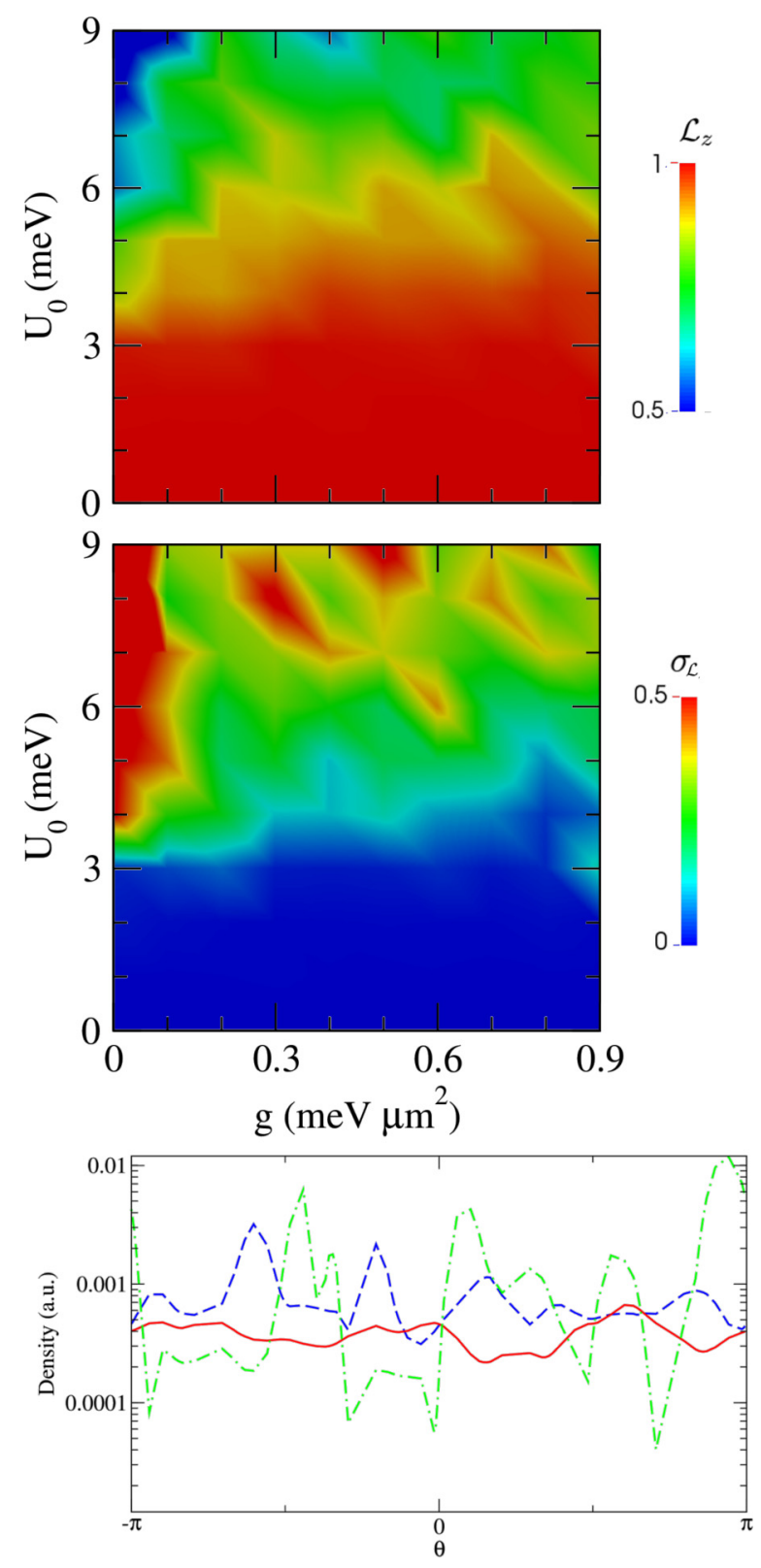

FIG. 3. Top panel: magnitude of the angular momentum $\mathcal{L}_{z}$ (normalized to its maximum value) as a function of the interaction $g$ and the disorder strength $U_{0}$. The value represented in the color map is the result of the average of ten dynamical simulations of the driven-dissipative Gross-Pitaevskii equation. Middle panel: standard deviation of $\mathcal{L}_{z}$ in the numerical calculations. The bottom panel represents the density profile of the $\sigma^{-}$component (in arbitrary units) along the ring at $r=R_{0}$ for different values of the interaction constant $g$ and disorder strength $U_{0}: g=0 \mathrm{meV} \mu \mathrm{m}^{2}$ and $U_{0}=$ $5 \mathrm{meV}$ (dot-dashed green line), $g=0.6 \mathrm{meV} \mu \mathrm{m}^{2}$ and $U_{0}=5 \mathrm{meV}$ (solid red line), and $g=0.6 \mathrm{meV} \mu \mathrm{m}^{2}$ and $U_{0}=8 \mathrm{meV}$ (dashed blue line).

exists, $\mathcal{L}_{z}$ does not fluctuate much from one realization to the next. Whereas in the regime where the disorder and the interactions compete equally, $\mathcal{L}_{z}$ is highly dependent on the details of $V_{\text {dis }}(\vec{r})$, and the standard deviation increases.

The simulations show also that in the noninteracting regime, density hot spots build up as a result of Anderson 
localization, and the current along the ring is thus substantially reduced. An example of this regime is shown in the bottom panel of Fig. 3 where the density profile at $r=R_{0}$ is shown for $g=0$ and $U_{0}=5 \mathrm{meV}$ (dot-dashed green line). Then, upon increasing the interactions to $g=0.6 \mathrm{meV} \mu \mathrm{m}^{2}$, the flow is almost restored and the polariton density becomes much more homogeneous within the ring (solid red line). This flow can be suppressed again by increasing the disorder amplitude to $U_{0}=8 \mathrm{meV}$. In this case, the polariton density exhibits other hot spots (dashed blue line).

\section{CONCLUSIONS}

In conclusion, in this work we have demonstrated the generation of superfluid currents with arbitrary winding number in a two-component polariton condensate by the mechanism of spin-to-orbital angular momentum conversion. This allows us to generate with a single pump two supercurrent states, differing by two units of winding number. Furthermore, we have studied the effect of a possible disorder on the currents. We have identified two main regimes at weak interactions, one where the polariton condensate is superfluid and screens the effect of disorder, and another where localization effects overcome superfluidity and strongly reduce the amplitude of the supercurrents. The latter regime is expected to occur for very large values of disorder strength or weak interactions. This allows us to conclude that one can expect robust superfluid current states under typical experimental conditions.

It is worth mentioning that large interactions may also lead to localization, as already found in one-dimensional bosonic and fermionic fluids [32]. This effect, however, occurs for much stronger values of interactions, where the mean-field approximation is not expected to hold. An interesting outlook would thus be to explore the interplay of superfluidity and interactions for bosons in drivendissipative conditions by going beyond the mean-field approximation. We would also like to see whether this configuration can be favorably exploited to manipulate the twocurrent condensate and study the dynamics of two-component superfluids.

\section{ACKNOWLEDGMENTS}

We acknowledge financial support from the Spanish MINECO (FIS2014-52285-C2-1-P and FIS2017-87801-P) and the European Regional development Fund, Generalitat de Catalunya Grants No. SGR2014-401 and No. SGR2017533. A.G. is supported by the Spanish MECD fellowship FPU13/02106, A.M. acknowledges funding from the ANR SuperRing (ANR-15-CE30-0012) and M.R. acknowledges funding from the ANR Quantum Fluids of Light (ANR-16CE30-0021).

\section{APPENDIX: SOAM CONVERSION IN HOMOGENEOUS TWO-DIMENSIONAL POLARITON GAS}

In this appendix we provide an alternative derivation of the SOAM conversion developed in Ref. [25] for the case of noninteracting polaritons in a homogeneous trap. We also suppose that the lifetime of the polaritons of both polarization components is equal. This is a realistic assumption, since the lifetime does not strongly depend on the polarization of the polariton condensate. Under this condition, we can write the coupled driven-dissipative Gross-Pitaevskii equations (1) in $\vec{k}$ space:

$$
i \hbar \frac{\partial}{\partial t} \Psi(\vec{k}, t)=[T(\vec{k})-i \hbar \gamma \mathbb{1}] \Psi(\vec{k}, t)+i E(\vec{k}, t),
$$

where $\Psi(\vec{k}, t)$ and $E(\vec{k}, t)$ are spinors that contain the polariton field of each polarization component of the polariton condensate, and the pump in each component, respectively. The kinetic tensor (2) can be written as:

$$
T(\vec{k})=\hbar\left(\begin{array}{cc}
\omega(\vec{k}) & \Delta(\vec{k}) e^{-i 2 \phi} \\
\Delta(\vec{k}) e^{i 2 \phi} & \omega(\vec{k})
\end{array}\right),
$$

where $k_{x}=k \cos \phi$ and $k_{y}=k \sin \phi$, and we have defined $2 \omega(\vec{k})=\omega_{\mathrm{TM}}(\vec{k})+\omega_{\mathrm{TE}}(\vec{k})$ and $2 \Delta(\vec{k})=\omega_{\mathrm{TM}}(\vec{k})-$ $\omega_{\mathrm{TE}}(\vec{k})$. We can write $T(\vec{k})-i \hbar \gamma \mathbb{1}$ in its diagonal form as $M D(\vec{k}) M^{-1}$, where $D(\vec{k})$ is the diagonal matrix whose elements are the eigenvalues of $T(\vec{k})-i \hbar \gamma \mathbb{1}: \hbar(\omega(\vec{k}) \pm$ $\Delta(\vec{k})-i \gamma)$, and $M$ is the change of basis matrix.

At this point, we can rewrite the spinor field and the pump as $\Psi(\vec{k}, t)=M \Phi(\vec{k}, t)$ and $E(\vec{k}, t)=M G(\vec{k}, t)$. Since $M$ is the matrix that diagonalizes the kinetic tensor, this transformation allows us to decouple the previous system of linear equations:

$$
i \hbar \frac{\partial}{\partial t} \Phi(\vec{k}, t)=D(\vec{k}) \Phi(\vec{k}, t)+i G(\vec{k}, t) .
$$

The solution of the homogenous part is

$$
\Phi_{H}(\vec{k}, t)=\Phi_{0}(\vec{k}) \exp (-i D(\vec{k}) t / \hbar),
$$

where $\Phi_{0}(\vec{k})$ is an initial condition for the polariton field, and the solution of the inhomogeneous part is:

$$
\Phi_{I}(\vec{k}, t)=\Phi_{H}(\vec{k}, t) \int_{0}^{t} \frac{G\left(\vec{k}, t^{\prime}\right)}{\hbar \Phi_{H}\left(\vec{k}, t^{\prime}\right)} d t^{\prime},
$$

with $\Phi(\vec{k}, t)=\Phi_{H}(\vec{k}, t)+\Phi_{I}(\vec{k}, t)$. The solution for $\Psi(\vec{k}, t)$ is then:

$$
\begin{aligned}
\Psi(\vec{k}, t)= & M \Phi_{H}(\vec{k}, t)+M \Phi_{I}(\vec{k}, t) \\
= & M \Phi_{0}(\vec{k}) \exp (-i D(\vec{k}) t / \hbar) \\
& +M \exp (-i D(\vec{k}) t / \hbar) \Phi_{0}(\vec{k}) \\
& \times \int_{0}^{t} \Phi_{0}(\vec{k})^{-1} \exp \left(i D(\vec{k}) t^{\prime} / \hbar\right) M^{-1} E(\vec{k}, t) d t^{\prime} .
\end{aligned}
$$

Due to the presence of the dissipative terms, which remain in the diagonal part of $D(\vec{k})$, one can see that the first term vanishes at long times. The second term of the sum simplifies as:

$$
\begin{aligned}
\Psi(\vec{k}, t) & =\int_{0}^{t} M \exp \left(-i D(\vec{k})\left(t-t^{\prime}\right) / \hbar\right) M^{-1} E(\vec{k}, t) d t^{\prime} \\
& =\int_{0}^{t} U\left(\vec{k}, t-t^{\prime}\right) \exp (-\gamma t) E\left(\vec{k}, t^{\prime}\right) d t^{\prime},
\end{aligned}
$$


where $U(\vec{k}, t)=e^{-i T(\vec{k}) t}$ is the time evolution operator corresponding to the kinetic tensor, which can be shown to be:

$$
U(\vec{k}, t)=e^{i \omega(\vec{k}) t}\left(\begin{array}{cc}
\cos (\Delta(\vec{k}) t) & i \exp (-i 2 \phi) \sin (\Delta(\vec{k}) t) \\
i \exp (i 2 \phi) \sin (\Delta(\vec{k}) t) & \cos (\Delta(\vec{k}) t)
\end{array}\right) .
$$

When the system is pumping only one of the components of the $\sigma^{+}-\sigma^{-}$basis, the spinor corresponding to the pump will be $E(\vec{k}, t)=f(\vec{k}, t)(1,0)^{T}$. With the aim of demonstrating the spin-to-orbital angular momentum effect, we will restrict the pump to the following shape: $f(\vec{k}, t)=f_{0} \delta\left(k-k_{p}\right) \delta(t)$. The polariton field in $\vec{k}$ can be calculated as:

$$
\left(\begin{array}{l}
\Psi_{+}(\vec{k}, t) \\
\Psi_{-}(\vec{k}, t)
\end{array}\right)=f_{0} \exp ((i \omega(\vec{k})-\gamma) t) \delta\left(k-k_{p}\right)\left(\begin{array}{c}
\cos (\Delta(\vec{k}) t) \\
i \exp (i 2 \phi) \sin (\Delta(\vec{k}) t)
\end{array}\right) .
$$

The corresponding polariton field in the real space is the inverse Fourier transform.

$$
\begin{aligned}
\left(\begin{array}{l}
\Psi_{+}(\vec{r}, t) \\
\Psi_{-}(\vec{r}, t)
\end{array}\right) & =f_{0} \exp (-i \gamma t) \int_{0}^{2 \pi} d \phi \int_{0}^{\infty} k d k \delta\left(k-k_{p}\right) \exp (i \omega(\vec{k}) t) \exp (i \vec{k} \cdot \vec{r})\left(\begin{array}{c}
\cos (\Delta(\vec{k}) t) \\
i \exp (i 2 \phi) \sin (\Delta(\vec{k}) t)
\end{array}\right) \\
& =f_{0} k_{p} \exp \left(\left(i \omega\left(k_{p}\right)-\gamma\right) t\right) \int_{0}^{2 \pi} d \phi \exp \left(i k_{p} r \cos (\theta-\phi)\right)\left(\begin{array}{c}
\cos \left(\Delta\left(k_{p}\right) t\right) \\
i \exp (i 2 \phi) \sin \left(\Delta\left(k_{p}\right) t\right)
\end{array}\right),
\end{aligned}
$$

where we have used that $\vec{k} \cdot \vec{r}=k r \cos (\theta-\phi)$, being $\theta$ and $\phi$ the orientation angles of $\vec{r}$ and $\vec{k}$, respectively. In order to solve the two integrals (one for each component), the following property of Bessel functions will be useful:

$$
\mathcal{J}_{n}(\zeta)=\frac{1}{2 \pi} \int_{-\pi}^{\pi} \exp (i(n \tau+\zeta \sin \tau)) d \tau
$$

The final solution is then:

$$
\left(\begin{array}{l}
\Psi_{+}(\vec{r}, t) \\
\Psi_{-}(\vec{r}, t)
\end{array}\right)=2 \pi f_{0} k_{p} \exp \left(\left(i \omega\left(k_{p}\right)-\gamma\right) t\right)\left(\begin{array}{c}
\mathcal{J}_{0}\left(k_{p} r\right) \cos \left(\Delta\left(k_{p}\right) t\right) \\
-i \mathcal{J}_{2}\left(k_{p} r\right) \exp (i 2 \theta) \sin \left(\Delta\left(k_{p}\right) t\right)
\end{array}\right) .
$$

We can see from the previous equation that when we pump one of the components, the polariton field of the other component acquires a phase pattern with a winding number 2 , which is a doubly-quantized vortex. This phenomenon has been already theoretically predicted in Ref. [25], and experimentally observed in Ref. [10], in the case of nontrapped polariton condensates.

It is worth commenting on the case where instead of pumping at a given modulus of $\vec{k}$ for all the possible angles in momentum space $\phi$, the orientation of $\vec{k}$ is also fixed. In this case, a term $\delta\left(\phi-\phi_{0}\right)$, where $\phi_{0}$ is the orientation direction of the pump wave vector, should be added to the pump. Then, the integral on $\phi$ when doing the inverse Fourier transform becomes trivial, and the solution is:

$$
\left(\begin{array}{l}
\Psi_{+}(\vec{r}, t) \\
\Psi_{-}(\vec{r}, t)
\end{array}\right)=2 \pi f_{0} k_{p} \exp \left(\left(i \omega\left(k_{p}\right)-\gamma\right) t\right) \exp \left(i k_{p} r \cos \left(\theta-\phi_{0}\right)\right)\left(\begin{array}{c}
\cos \left(\Delta\left(k_{p}\right) t\right) \\
-i \exp \left(i 2 \phi_{0}\right) \sin \left(\Delta\left(k_{p}\right) t\right)
\end{array}\right) .
$$

The previous solution does not content any vortex profile, hence, in order to nucleate a vortex, it is crucial not to fix the wave vector orientation and excite all the possible angles in momentum space. As an example, if we pump with the pump wave vector oriented along the $x$ direction, the phase pattern of the minority component will be the one of a plane wave traveling in the $x$ direction.

[1] J. File and R. G. Mills, Phys. Rev. Lett. 10, 93 (1963).

[2] J. D. Reppy and D. Depatie, Phys. Rev. Lett. 12, 187 (1964).

[3] A. Ramanathan, K. C. Wright, S. R. Muniz, M. Zelan, W. T. Hill, C. J. Lobb, K. Helmerson, W. D. Phillips, and G. K. Campbell, Phys. Rev. Lett. 106, 130401 (2011).

[4] A. Amo, J. Lefrère, S. Pigeon, C. Adrados, C. Ciuti, I. Carusotto, R. Houdré, E. Giacobino, and A. Bramati, Nat. Phys. 5, 805 (2009).

[5] K. G. Lagoudakis, M. Wouters, M. Richard, A. Baas, I. Carusotto, R. André, L. S. Dang, and B. Deveaud-Plédran, Nat. Phys. 4, 706 (2008).

[6] K. G. Lagoudakis, T. Ostatnický, A. V. Kavokin, Y. G. Rubo, R. André, and B. Deveaud-Plédran, Science 326, 974 (2009).
[7] D. Sanvitto, F. M. Marchetti, M. H. Szymanska, G. Tosi, M. Baudisch, F. P. Laussy, D. N. Krizhanovskii, M. S. Skolnick, L. Marrucci, A. Lemaître et al., Nat. Phys. 6, 527 (2010).

[8] V. Kohnle, Y. Léger, M. Wouters, M. Richard, M. T. PortellaOberli, and B. Deveaud-Plédran, Phys. Rev. Lett. 106, 255302 (2011).

[9] T. Boulier, E. Cancellieri, N. D. Sangouard, Q. Glorieux, A. V. Kavokin, D. M. Whittaker, E. Giacobino, and A. Bramati, Phys. Rev. Lett. 116, 116402 (2016).

[10] F. Manni, K. G. Lagoudakis, T. K. Paraïso, R. Cerna, Y. Léger, T. C. H. Liew, I. A. Shelykh, A. V. Kavokin, F. Morier-Genoud, and B. Deveaud-Plédran, Phys. Rev. B 83, 241307(R) (2011).

[11] T. Jacqmin, I. Carusotto, I. Sagnes, M. Abbarchi, D. D. Solnyshkov, G. Malpuech, E. Galopin, A. Lemaître, J. Bloch, and A. Amo, Phys. Rev. Lett. 112, 116402 (2014). 
[12] F. Marsault, H. S. Nguyen, D. Tanese, A. Lemaître, E. Galopin, I. Sagnes, A. Amo, and J. Bloch, Appl. Phys. Lett. 107, 201115 (2015).

[13] N. Y. Kim, K. Kusudo, C. Wu, N. Masumoto, A. Löffler, S. Höfling, N. Kumada, L. Worschech, A. Forchel, and Y. Yamamoto, Nat. Phys. 7, 681 (2011).

[14] P. W. Anderson, Phys. Rev. 109, 1492 (1958).

[15] D. M. Basko, I. L. Aleiner, and B. L. Altshuler, Ann. Phys. 321, 1126 (2006).

[16] D. M. Basko, I. L. Aleiner, and B. L. Altshuler, Phys. Rev. B 76, 052203 (2007).

[17] A. Baas, K. G. Lagoudakis, M. Richard, R. André, L. S. Dang, and B. Deveaud-Plédran, Phys. Rev. Lett. 100, 170401 (2008).

[18] B. Deissler, M. Zaccanti, G. Roati, C. D’Errico, M. Fattori, M. Modugno, G. Modugno, and M. Inguscio, Nat. Phys. 6, 354 (2010).

[19] G. Modugno, Rep. Prog. Phys. 73, 102401 (2010).

[20] A. C. Bleszynski-Jayich, W. E. Shanks, B. Peaudecerf, E. Ginossar, F. von Oppen, L. Glazman, and J. G. E. Harris, Science 326, 272 (2009).

[21] M. Filippone, P. W. Brouwer, J. Eisert, and F. von Oppen, Phys. Rev. B 94, 201112 (2016).
[22] I. Carusotto and C. Ciuti, Rev. Mod. Phys. 85, 299 (2013).

[23] G. Panzarini, L. C. Andreani, A. Armitage, D. Baxter, M. S. Skolnick, V. N. Astratov, J. S. Roberts, A. V. Kavokin, M. R. Vladimirova, and M. A. Kaliteevski, Phys. Rev. B 59, 5082 (1999).

[24] K. V. Kavokin, I. A. Shelykh, A. V. Kavokin, G. Malpuech, and P. Bigenwald, Phys. Rev. Lett. 92, 017401 (2004).

[25] T. C. H. Liew, A. V. Kavokin, and I. A. Shelykh, Phys. Rev. B 75, 241301 (2007).

[26] G. Carleo, G. Boéris, M. Holzmann, and L. Sanchez-Palencia, Phys. Rev. Lett. 111, 050406 (2013).

[27] D. Delande and G. Orso, Phys. Rev. Lett. 113, 060601 (2014).

[28] P. Renucci, T. Amand, X. Marie, P. Senellart, J. Bloch, B. Sermage, and K. V. Kavokin, Phys. Rev. B 72, 075317 (2005).

[29] M. Vladimirova, S. Cronenberger, D. Scalbert, K. V. Kavokin, A. Miard, A. Lemaître, J. Bloch, D. Solnyshkov, G. Malpuech, and A. V. Kavokin, Phys. Rev. B 82, 075301 (2010).

[30] A. V. Sekretenko, S. S. Gavrilov, and V. D. Kulakovskii, Phys. Rev. B 88, 195302 (2013).

[31] N. Takemura, S. Trebaol, M. Wouters, M. T. Portella-Oberli, and B. Deveaud, Phys. Rev. B 90, 195307 (2014).

[32] T. Giamarchi and H. J. Schulz, Phys. Rev. B 37, 325 (1988). 